\title{
Beating the shot-noise limit
}

\author{
Avraham Gover $^{1 \star}$, Ariel Nause ${ }^{1}$, Egor Dyunin $^{1}$ and Mikhail Fedurin ${ }^{2}$
}

The current shot-noise of an electron beam is proportional to its average current and the frequency bandwidth. This is a consequence of the Poisson distribution statistics of particles emitted at random from any source. Here we demonstrate noise suppression below the shot-noise limit in optical frequencies for relativistic electron beams. This process is made possible by collective Coulomb interaction between the electrons of a cold intense beam during beam drift'. The effect was demonstrated by measuring a reduction in optical transition radiation power per unit of electron-beam pulse charge. This finding indicates that the beam charge homogenizes owing to the collective interaction, and its distribution becomes subPoissonian. The spontaneous radiation emission from such a beam would also be suppressed (Dicke's subradiance ${ }^{2}$ ). Therefore, the incoherent spontaneous radiation power of any electron-beam radiation source (such as free-electron lasers ${ }^{3,4}$ ) can be suppressed, and the classical coherence limits ${ }^{5}$ of seed-injected free-electron lasers 6 may be surpassed.

Current shot-noise suppression in an electron beam in the optical frequency regime is an effect of particle self-ordering and charge homogenization on the scale of optical wavelengths ${ }^{7}$, which statistically corresponds to the exhibition of sub-Poissonian electron number statistics ( similarly to photons in squeezed light ${ }^{8}$ ). A dispute over the feasibility of this effect at optical frequencies is resolved in the experiment reported here. We have evidence for electron-beam noise suppression from measurement of the optical transition radiation (OTR) power emitted by a beam on incidence on a metal screen after passing through a drift section. The OTR emission is proportional to the current shot-noise of the incident beam.

In a randomly distributed stream of particles that satisfies Poisson statistics, the variance of the number of particles that pass through any cross-section at any time period $T$ is equal to the number of particles $N_{T}$ that pass this cross-section during the same time $T$, averaged over different times of measurements. Consequently, the current fluctuation is $I=e \sqrt{N_{T}} / T=e \sqrt{I_{\mathrm{b}} T / e} / T=\sqrt{e I_{\mathrm{b}} / T}$. When formally calculated, the average beam shot-noise spectral power $(-\infty<\omega<\infty)$ is:

$$
\overline{|\check{I}(\omega)|^{2}}=e I_{\mathrm{b}}
$$

Here $I(t)$ is the beam current modulation, and the Fourier transform is defined as $\check{I}(\omega)=\int_{-\infty}^{\infty} \mathrm{e}^{i \omega t} I(t) \mathrm{d} t$.

In radiofrequency linear accelerators (RF-LINACs) it is usually assumed that collective inter-particle interaction is negligible during beam acceleration and transport, and the shot-noise limit (1) applies. However, with recent technological advances in radiofrequency accelerators, and in particular the development of photocathode guns ${ }^{9}$, high-quality cold and intense accelerated electron beams are available, and the neglect of collective microdynamic interaction effects in the transport of such electron beams is no longer justified. Effects of coherent OTR emission and super-linear scaling with $I_{\mathrm{b}}$ of the OTR emission intensity were observed in the LCLS (LINAC Coherence Light Source) injector ${ }^{10}$ at SLAC and in other laboratories ${ }^{11}$. These effects, originally referred to as unexpected physics ${ }^{12}$, are now clearly recognized as the result of collective Coulomb micro-dynamic interaction and establishment of phase correlation between the electrons in the beam. In these cases, however, the collective interaction led to shot-noise and OTR power enhancement (gain), and not to suppression. Collective effects were shown to be responsible also for beam instabilities (micro-bunching instability) that were observed in dispersive electron-beam transport elements ${ }^{13,14}$. Collective microdynamic evolution of $1 \mathrm{THz}$ coherent singlefrequency current modulation was recently reported ${ }^{15}$, but no stochastic optical noise suppression effect could be observed.

Noise gain due to collective interaction has been demonstrated in numerous laboratories, but the notion of beam noise suppression at optical frequencies ${ }^{1}$ has been controversial (although analogous effects were known in non-relativistic microwave tubes ${ }^{16}$ ). To explain the physics of the noise suppression, we point out that in the electron-beam frame of reference the effect of noise suppression appears as charge density homogenization. The simple argument that follows shows that the space-charge force, which is directed to expand higher density charge bunches, has a dominant effect over the randomly directed Coulomb repulsion force between the particles (Fig. 1).

Assume that in some regions of the beam, there is higher particle-density bunching. Encompassing such a bunch within a sphere of diameter $d^{\prime}$, the excess charge in this sphere is $e \Delta N^{\prime}$, and the potential energy of an electron on the surface of the sphere is $\varepsilon_{\text {sc }}=e^{2} \Delta N^{\prime} / 2 \pi \varepsilon_{0} d^{\prime}$. This potential energy turns in time into kinetic energy of electrons, transferred in the direction of bunch expansion (homogenization). At the same time, the electron also possesses an average potential energy due to the Coulomb interaction with neighbour electrons at an average distance $n_{0}^{\prime-1 / 3}$ ( $n_{0}^{\prime}$ is the average density in the beam frame). This potential energy $\varepsilon_{\text {Coul }}=e^{2} / 4 \pi \varepsilon_{0} n_{0}^{\prime-1 / 3}$ turns into kinetic energy of electrons that are accelerated in random directions. In a randomly distributed electron beam that satisfies Poisson statistics $\Delta N^{\prime}=N^{\prime 1 / 2}$, where $N^{\prime}=\pi d^{\prime 3} n_{0}^{\prime} / 6$ is the average number of electrons in such a sphere. Therefore, the condition for domination of the directed spacecharge energy over the random energy is

$$
\frac{\varepsilon_{\mathrm{sc}}}{\varepsilon_{\mathrm{Coul}}}=\left(\frac{2 \pi}{3} \frac{d^{\prime}}{n_{0}^{\prime-1 / 3}}\right)^{1 / 2}>1
$$

We conclude that a random cold beam of density $n_{0}^{\prime}$ always tends to homogenize in any spatial scale larger than the average inter-particle distance: $d^{\prime}>n_{0}^{\prime-1 / 3}$.

The expansion trend of a dense bunch would clearly tend to homogenize the charge distribution of an initially cold electron-beam plasma. The timescale for the homogenization process can be connected only to the plasma frequency-the

\footnotetext{
${ }^{1}$ Tel-Aviv University, Tel-Aviv 69978, Israel, ${ }^{2}$ ATF, Brookhaven National Lab, Upton, New York 11973, USA. *e-mail: gover@eng.tau.ac.il.
} 


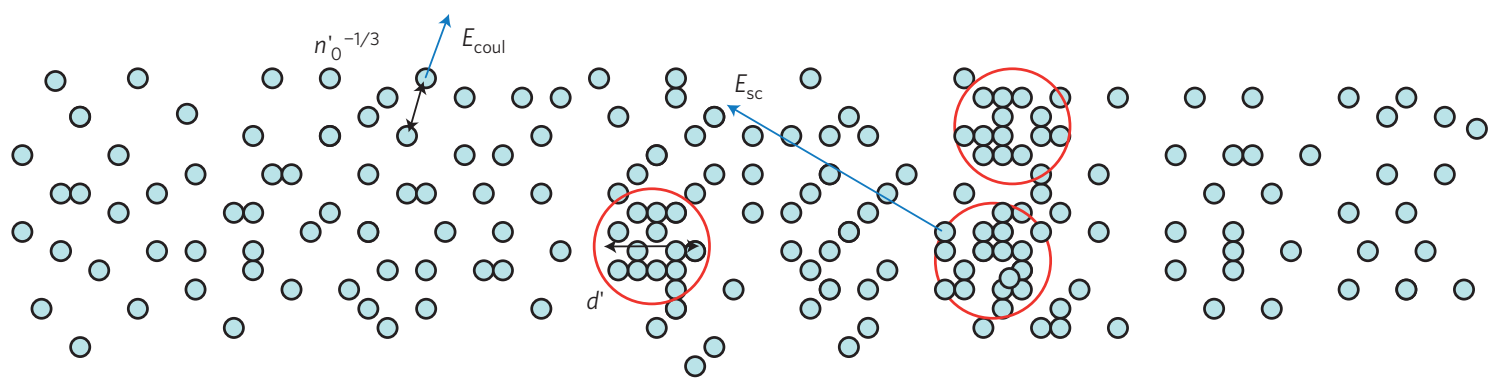

Figure 1 | Random distribution in an electron beam, as viewed in the beam reference frame. The expansion-directed space-charge force $\left(-\mathrm{e} E_{\mathrm{sc}}\right)$, exerted on electrons on the boundaries of a higher density bunch, exceeds the random inter-particle Coulomb force ( $\left.-\mathrm{e} E_{\text {Coul }}\right)$.

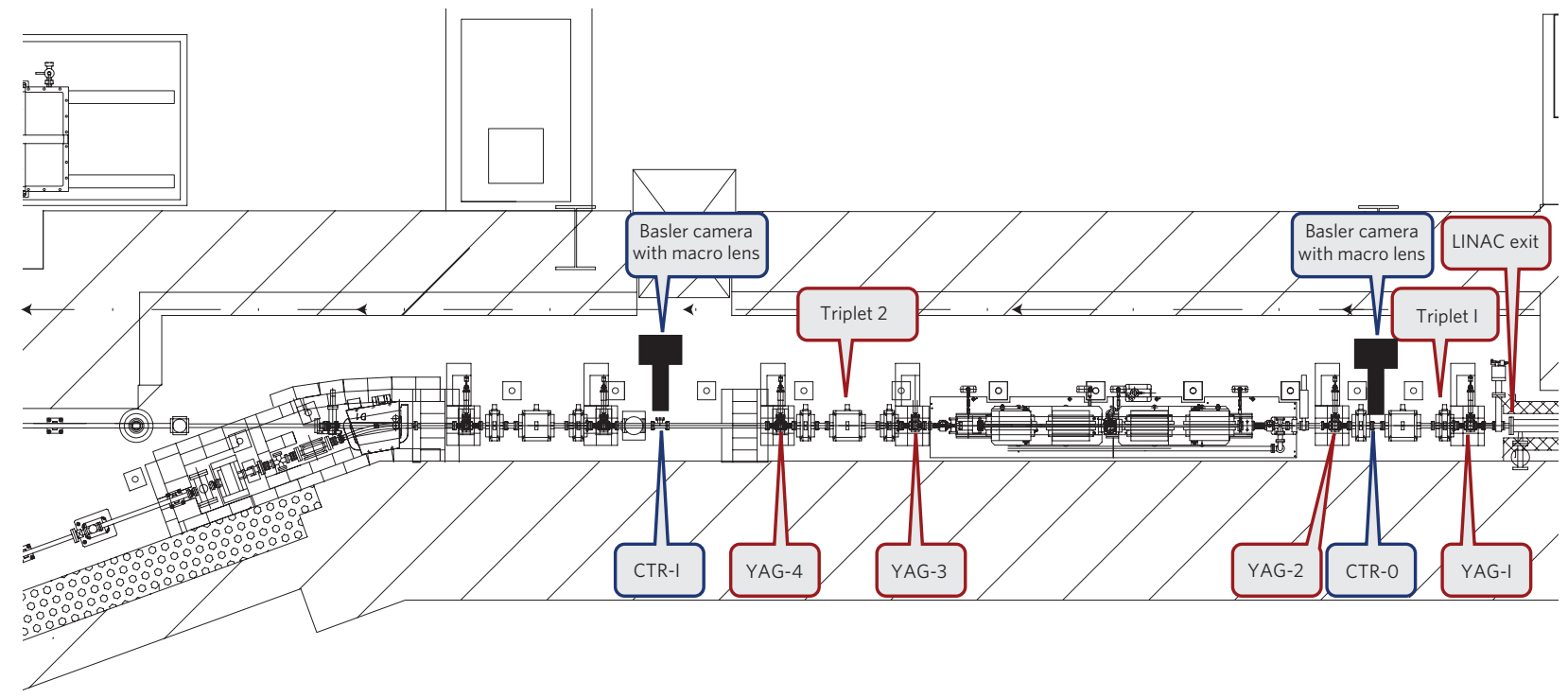

Figure 2 | The experimental set-up. The ATF electron-beam transport set-up is shown from the injector RF-LINAC exit to the OTR viewer site (CTR-1). The accelerated beam energy was varied from 50 to $70 \mathrm{MeV}$ and the beam charge was varied from 200 to $500 \mathrm{pC}$. The beam spot dimensions were measured at four locations using fluorescent screens (YAG-1 to YAG-4). The beam envelope was kept nearly uniform, except in the section between quadruple lenses TRIPLET-1 and TRIPLET-2 where it was focused to a narrow waist in the centre of the free drift section between the triplets (the chicane in the drawing was turned off). An electronic signal proportional to the photon number of integrated OTR emission from CTR-1 was measured using a CCD camera. Independently, a similar reference measurement was carried out at CTR-O before the collective interaction drift region.

fundamental collective excitation in the beam, namely: $\sim \pi / \omega_{\mathrm{p} 0}^{\prime}$, where $\omega_{\mathrm{p} 0}^{\prime}=\left(e^{2} n_{0}^{\prime} / m \varepsilon_{0}\right)^{1 / 2}$. Interestingly, this is the characteristic expansion time of a bunch of charged particles as can be verified by calculating or simulating the expansion time of a sphere or disc of charged particles (see Supplementary Appendices S1 and S2).

In electron-beam transport under appreciable space-charge conditions, the microdynamic noise evolution process may be viewed as the stochastic oscillations of Langmuir plasma waves ${ }^{1}$. In the linear regime, the evolution of longitudinal current and velocity modulations of a beam of average current $I_{\mathrm{b}}$, velocity $\beta c$ and energy $E=(\gamma-1) m c^{2}$, can be described in the laboratory frame by ${ }^{17}$ :

$$
\begin{aligned}
& \frac{\mathrm{d}}{\mathrm{d} \varphi_{\mathrm{p}}} \check{\iota}(\omega, z)=-\frac{i}{W(z)} \check{v}(\omega, z) \\
& \frac{\mathrm{d}}{\mathrm{d} \varphi_{\mathrm{p}}} \check{v}(\omega, z)=-i W(z) \check{\iota}(\omega, z)
\end{aligned}
$$

where $\check{l}(\omega)=\check{I}(\omega) \mathrm{e}^{i \omega z / \beta c}, \check{v}(\omega)=\check{V}(\omega) \mathrm{e}^{i \omega z / \beta c}, \check{I}(\omega), \check{V}(\omega)$ are the respective Fourier components of the beam current and kineticvoltage modulations. The kinetic-voltage modulation is related to

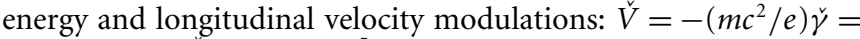
$-\left(m c^{2} / e\right) \gamma^{3} \beta \tilde{\beta} ; \varphi_{\mathrm{p}}(z)=\int_{0}^{z} \theta_{\mathrm{pr}}\left(z^{\prime}\right) \mathrm{d} z^{\prime}$ is the accumulated plasma phase; $W(z)=r_{\mathrm{p}}^{2} /\left(\omega A_{e} \theta_{\mathrm{pr}} \varepsilon_{0}\right)$ is the beam wave-impedance; $A_{e}$ is the effective beam cross-section area, $\theta_{\mathrm{pr}}=r_{\mathrm{p}} \omega_{\mathrm{pl}} / \beta c$ is the plasma wavenumber of the Langmuir mode; $r_{\mathrm{p}}<1$ is the plasma reduction factor; $\omega_{\mathrm{pl}}=\omega_{\mathrm{p} 0} / \gamma^{3 / 2}$ is the longitudinal plasma frequency in the laboratory frame.

The single-frequency Langmuir plasma wave model expression (3) can be solved straightforwardly in the case of uniform drift transport. After employing an averaging process, this results in a simple expression for the spectral parameters of stochastic current and velocity fluctuations (noise) in the beam assuming that they are initially uncorrelated ${ }^{1,17}$ :

$$
\overline{|\check{l}(\omega, L)|^{2}}=\overline{|\check{\iota}(\omega, 0)|^{2}} \cos ^{2} \varphi_{\mathrm{p}}(L)+\left(\overline{|\check{v}(\omega, 0)|^{2}} / W^{2}\right) \sin ^{2} \varphi_{\mathrm{p}}(L)
$$

The beam current noise evolution is affected by the initial axial velocity noise through the parameter $N^{2}=$ $\overline{|\check{v}(\omega, 0)|^{2}} / W^{2} \overline{|\check{l}(\omega, 0)|^{2}}=\left(\omega / c \beta k_{\mathrm{D}}\right)^{2}$, where $k_{\mathrm{D}}=\omega_{\mathrm{pl}} / c \sigma_{\beta}$ is the Debye wavenumber and $c \sigma_{\beta}$ is the axial velocity spread. Equation (4) suggests that current noise suppression is possible if the beam is initially cold $-N^{2}<1$, and if plasma phase accumulation of $\varphi_{\mathrm{p}}(L) \sim \pi / 2$ is feasible. This condition also assures that Landau damping is negligible ${ }^{18}$.

The noise suppression demonstration experiment was conducted on the $70 \mathrm{MeV}$ RF-LINAC of ATF/BNL (Fig. 2). The beam current noise measurement was made by recording the 


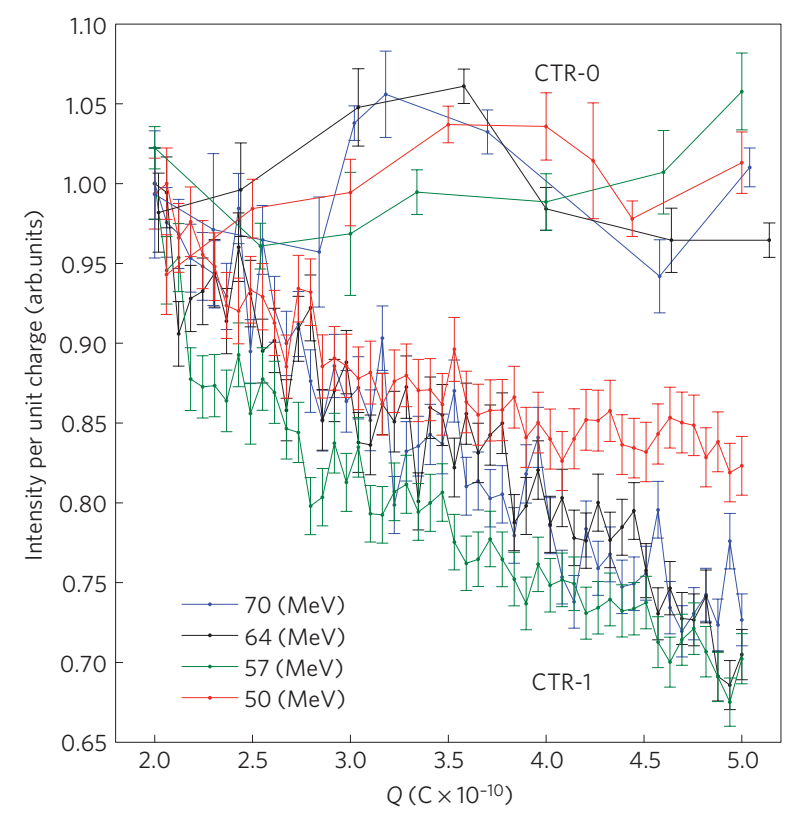

Figure 3 | Integrated OTR intensity measurement signals divided by the electron pulse charge. The CTR-1 data correspond to OTR measurement from a screen, intercepting the electron beam $6.5 \mathrm{~m}$ away from the LINAC exit. The curves' negative slope indicates relative current shot-noise suppression of $20-30 \%$ as the beam charge varies from 0.2 to $0.5 \mathrm{nC}$ at different beam energies (50-70 MeV). The CTR-0 data correspond to OTR measurement right after the LINAC exit. Used for reference, its horizontal slope (linear scaling of $S_{\text {OTR }}$ with beam charge) indicates that there was no charge suppression before the collective microdynamic process in the subsequent drift section. The error of the camera integrated signal measurement, $3 \%$, was determined from the variance in the value of the signal due to pulse-to-pulse variation, measured repeatedly while keeping all beam control parameters fixed. The charge measurement error is $1 \%$.

OTR radiation emitted from a copper screen placed $L=6.5 \mathrm{~m}$ away from the accelerator exit. Keeping the camera and screen CTR-1 position fixed, the practical way to control the plasma phase $\varphi_{\mathrm{p}}$ was to vary the beam pulse charge $(200-500 \mathrm{pC}$ ) (as in ref. 15$)$ and the beam energy $E=(\gamma-1) m c^{2}(50-70 \mathrm{MeV})$. The quad settings were readjusted for each beam acceleration energy, and the beam spot sizes $\sigma_{x}, \sigma_{y}$ were measured at four points (YAG1-YAG4) along the beam transport line (Fig. 2). For reference, the OTR signal was measured independently also on a screen CTR-0 preceding the drift section.

The measured signal $S_{\text {OTR }}$ was the integrated charge accumulated in all of the pixels of a CCD (charge-coupled device) camera exposed to the OTR emission on the incidence of single microbunch electron-beam pulses on the screen. The measured data of $S_{\mathrm{OTR}} / Q_{\mathrm{b}}$ in CTR-0 and CTR-1 are shown in Fig. 3 as a function of the varied bunch charge $Q_{b}$ in the range 200-500 pC. The pulse duration in all experiments remained approximately the same (5 pS) corresponding to an average current of 40-100 A (see Supplementary Information). In a shot-noise-dominated beam, in the absence of collective microdynamics, the current noise and consequently $S_{\mathrm{OTR}}$ are proportional to $I_{\mathrm{b}}(1)$. Our measured data of $S_{\mathrm{OTR}} / Q_{\mathrm{b}}$ in CTR-0 lie approximately on a horizontal line, confirming the absence of collective microbunching and current-velocity noise correlation or noise suppression before this point. On the other hand, the data measured on CTR-1 exhibit a systematic drop as a function of charge (200-500 pC) for all beam energies (50-70 MeV). As the measurement conditions at the two measurement positions and at different beam energies could not be made identical, the absolute suppression level between the two points could not be determined. However, the normalized data of all measurements of noise per unit charge depicted in Fig. 3 show the scaling with charge for all beam energies. This demonstrates attainment of $20-30 \%$ relative noise suppression, and confirms the predicted effect of collective microdynamic noise suppression process in the drift section.

Beyond observation of noise suppression, interpreting the measured data and the noise suppression rate in terms of a simple theoretical model as presented by equation (4) would be very crude. For $N^{2} \ll 1$ equation (4) predicts maximum suppression by a factor $N^{2}$ at $\varphi_{\mathrm{p}}=\pi / 2$, but this is true only for uniform beam transport. In the present transport configuration the beam was focused to a tight waist in the section between triplet 1 and triplet 2 (the location of a turned-off chicane). According to a theorem of ref. 1 a plasma phase increment $\varphi_{\mathrm{p}}=\pi / 2$ is accumulated along a beam waist, but this applies only if the beam transport is fully space-charge dominated. This is not the case for the present experiment conditions in which the beam angular spread due to emittance and beam focusing is significant, and the collective microdynamic noise suppression process is compromised. Solving the more general equation (3) under conditions of varying beam cross-section and increased angular spread (the focusing at triplet 1 at different beam energies led to rather high values of $N^{2}$ : $0.3-0.8$ ) shows that the noise suppression expected in a model configuration similar to the present experimental configuration is substantially smaller than anticipated in the uniform beam model (4) (see Supplementary Appendix S4). This model calculation also shows that the noticeable weak dependence of the relative suppression rate on the beam energy observed in Fig. 3 is consistent with calculations at the experimental conditions: the downscaling of $\omega_{\mathrm{pl}}$ with $\gamma^{3 / 2}$ is offset by the increased current density at the focused beam waist at higher beam energies. This weak energy dependence is consistent with a point of view that in the beam rest frame the beam envelope expansion and the charge homogenization effects are related ${ }^{1}$. Other factors that can reduce the collective microdynamic suppression rate may be three-dimensional effects and excitation of higher-order Langmuir plasma waves of different wavenumber values $\theta_{\mathrm{pr}}($ refs 7,19$)$. These and other deviations from ideal conditions can explain why the relative noise suppression effect measured in this experiment in the range of charge variation (200-500 pC) is quite modest (20-30\%).

The observation of current shot-noise suppression at optical frequencies, although small, is of interest from the point of view of both fundamental physics and of applications points of view. The electron-beam shot-noise expression (1) is a direct consequence of the Poisson statistics of random particle number distribution, and therefore is widely considered to be an absolute limit. The experiment demonstrates that at least at optical frequencies this limit may be surpassed in charged particles owing to Coulomb collective interaction. The suppression of OTR emission is also a noteworthy demonstration of a fundamental physical process, as it is a vivid demonstration of spontaneous emission subradiance in Dicke's sense $e^{2}$ in the classical limit. This subradiance is attributable to the more uniform rate of incidence of electrons on the OTR screen after the beam charge is homogenized. This same process would be expected also to suppress spontaneous and self-amplified spontaneous emission in any free-electron radiation device, including undulators and free-electron lasers (ref. 5), as was first suggested in ref. 20. Furthermore, microdynamic control over electron-beam noise may be also of practical use in particle beam physics, and help in controlling beam instability in the transport of intense high-quality beams.

Theory predicts that much bigger factors of shot-noise suppression are possible in more favourable configurations, and schemes have been suggested for producing the noise suppression process in shorter lengths using dispersive transport ${ }^{17,21}$ (dispersive transport 
noise suppression has just been demonstrated experimentally in the LCLS (ref. 22)). We have demonstrated noise suppression at optical frequencies-four orders of magnitude higher than the previous microwave noise suppression works. Further research and beam quality improvements are required to determine the short-wavelength limits of applicability of this process ${ }^{23}$, a limit that is ultimately bound at X-ray wavelengths by the beam charge granularity inter-particle spacing limit (2).

\section{Methods}

The experimental set-up is presented in Fig. 2. The electron beam, which was generated in a photocathode by ultrafast pulses $\left(t_{\text {pulse }}=5 \mathrm{pS}\right)$ of a Nd-YAG laser, was accelerated by an RF-LINAC to beam energies of 70, 64, 57 and $50 \mathrm{MeV}$. It was accelerated at the on-crest phase to avoid chirped energy variation along the beam.

The noise measurements were carried out by recording the integrated OTR radiation from the beam in screen CTR-1, positioned $6.5 \mathrm{~m}$ after the LINAC exit. The beam was allowed to drift freely between two quad triplets (QUAD-1, QUAD-2) that focused the beam and were readjusted for different beam energies. A reference OTR measurement was done in a separate setting (CTR-0), just after the LINAC exit, showing a lack of noise suppression effect at this point.

To control the collective microdynamic process we varied the plasma phase by varying the beam charge $Q_{\mathrm{b}}$ in 50 equal increments (in the reference measurement 8 increments) between 0.2 and $0.5 \mathrm{nC}$ in each of the four beam energy experiments. The photo-cathode current was varied by attenuating the incident laser beam using variable-angle crossed polarizers. This method made it possible to fix the OTR screen and camera at the same position in all experiments, and enabled stable comparative data collection in all experiments. There was no change in the beam spot dimensions as the beam charge was varied in the range 200-500 pC. As the acceleration energy was varied, the beam spots on the screens were readjusted and kept small by controlling the two quad triplets.

We used a (Basler) scA-1400 CCD camera equipped with a (Nikkor) macro lens $(100 \mathrm{~mm} \mathrm{~F} / \#=2.8)$ to obtain 1:1 image magnification of the OTR Copper screen (CTR-1). The screen was placed at $45^{\circ}$ to the beam line. The current noise measurement is based on its proportionality to the integrated OTR photon number, measured by integrating all of the pixels using a frame grabber. The camera aperture opening angle, operating in screen imaging mode, was wide enough to collect the entire OTR radiation lobe (of opening angle $\sim 4 / \gamma$ ) in all experiments. The photographed 1:1 image of the OTR spot on the $11 \mathrm{~mm}$ (diagonal size) CCD chip was small enough to assure full collection of photons in the measured wavelength range. As the optical spectrum of the electron-beam noise (and the OTR photons) is quite uniform in the spectral range of the camera sensitivity $(\lambda=0.4-1 \mu \mathrm{m})$, and the integrated OTR photon number is only weakly (logarithmically) dependent on the beam energy ${ }^{24}$, the proportionality factor between the measured integrated pixels charge, $S_{\text {OTR }}$, and the current noise power within the measured spectral range was nearly the same for all experiments.

The quad currents were varied as the beam acceleration energy was changed, to focus the beam within the drift section, and keep the beam spot well within the camera frame in all experiments. The beam spots on screens YAG-1 to YAG-4 were recorded. The measured spot dimensions at these points were used to evaluate the beam profile dimensions along the drift section at different acceleration energies, employing a three-dimensional numerical code simulation with space-charge effects (General Particles Tracer ${ }^{25}$ ).

Received 19 January 2012; accepted 7 September 2012; published online 14 October 2012

\section{References}

1. Gover, A. \& Dyunin, E. Collective interaction control of optical frequency shot-noise in charged particle beams. Phys. Rev. Lett. 102, 154801 (2009).

2. Dicke, R. H. Coherence in spontaneous radiation processes. Phys. Rev. 93, 99-110 (1954).

3. Emma, P. et al. First lasing and operation of an ångstrom-wavelength free-electron laser. Nature Photon. 4, 641-647 (2010).

4. Saldin, E. L., Schneidmiller, E. A. \& Yurkov, M. V. The Physics of Free Electron Lasers (Springer, 1999).
5. Gover, A. \& Dyunin, E. Coherence limits of free electron lasers. J. Quant. Electron. 46, 1511-1517 (2010).

6. Labat, M. et al. High-gain harmonic-generation free-electron laser seeded by harmonics generated in gas. Phys. Rev. Lett. 107, 224801 (2011).

7. Nause, A., Dyunin, E. \& Gover, A. Optical frequency shot- noise suppression in electron beams: 3D analysis. J. Appl. Phys 107, 103101 (2010).

8. Walls, D. F. Squeezed states of light. Nature 306, 141-146 (1983)

9. O'Shea, P. \& Freund, H. Free electron lasers: Status and applications. Science 292, 1853-1858 (2001).

10. Loos H. et al. Observation of coherent optical transition radiation in the LCLS LINAC Proc. FEL08 THBAU01 (2008).

11. Lumpkin, A. et al. First observations of COTR due to a microbunched beam in the VUV at $157 \mathrm{~mm}$. Nucl. Instrum. Methods Phys. Res. A 528, 194-198 (2004).

12. Akre, R. et al. Commissioning the linac coherent light source injector. Phys. Rev. ST-AB 11, 030703 (2008).

13. Shaftan, T. \& Huang, Z. Experimental characterization of a space charge induced modulation in high-brightness electron beam. Phys. Rev. ST-AB 7, 080702 (2004).

14. Huang, Z. et al. Suppression of microbunching instability in the linac coherent light source. Phys. Rev. ST-AB 7, 074401 (2004).

15. Musumeci, P., Li, R. K. \& Marinelli, A. Nonlinear longitudinal space charge oscillations in relativistic electron beams. Phys. Rev. Lett. 106, 184801 (2011)

16. Haus, H. A. \& Robinson, N. H. The minimum noise figure of microwave amplifiers. Proc. IRE 43, 981-991 (1955).

17. Gover, A., Dyunin, E., Duchovni, T. \& Nause, A. Collective microdynamics and noise suppression in dispersive electron beam transport. Phys. Plasmas 18, 123102 (2011).

18. Marinelli, A., Hemsing, E. \& Rosenzweig, J. B. Three dimensional analysis of longitudinal plasma oscillations in a thermal relativistic electron beam. Phys. Plasmas 18, 103105 (2011).

19. Venturini, M. Models of longitudinal space-charge impedance for microbunching instability. Phys. Rev. ST-AB 11, 034401 (2008).

20. Dyunin, E. \& Gover, A. The general velocity and current modulation linear transfer matrix of FEL and control over SASE power in the collective regime. Nucl. Instrum. Methods Phys. A593, 49-52 (2008).

21. Ratner, D., Huang, Z. \& Stupakov, G. Analysis of shot noise suppression for electron beams. Phys. Rev. ST-AB 14, 060710 (2011).

22. Ratner, D. \& Stupakov, G. Observation of Shot Noise suppression at optical wavelengths in a relativistic electron beam. Phys. Rev. Lett. 109, 034801 (2012).

23. Kim, K-J. \& Lindberg, R. R. Collective and individual aspects of fluctuations in relativistic electron beams for free electron lasers, Proc. FEL2011 TUOA2 (2011).

24. Ginzburg, V. L. Transition radiation and transition scattering. Phys. Scr. 2, 182-191 (1982)

25. GPT User Manual, Pulsar Physics, Flamingostraat 24, 3582 SX Utrecht, The Netherlands. http://www.pulsar.nl/gpt/index.html

\section{Acknowledgements}

We acknowledge advice and assistance in conducting the experiment from V. Yakimenko and the ATF team, and helpful discussions with T. Shaftan, P. Muggli, G. Andonian, Z. Schuss, A. Arie and A. Eyal. This work was supported in part by the Israel Science Foundation Grant No. 353/09 and by a Rahamimoff grant of the US-Israel BSF.

\section{Author contributions}

A.G. contributed the theoretical and experimental concept. A.N. contributed to the experimental conception, designed and performed the experiment, data analysis and numerical simulations. E.D. contributed to the theoretical analysis of the concept and the experiment. M.F. contributed to performing the experiment on the ATF accelerator.

\section{Additional information}

Supplementary information is available in the online version of the paper. Reprints and permissions information is available online at www.nature.com/reprints. Correspondence and requests for materials should be addressed to A.G.

\section{Competing financial interests}

The authors declare no competing financial interests. 